\title{
Learning Compact Model for Large-Scale Multi-Label Data
}

\author{
Tong Wei, Yu-Feng Li \\ National Key Laboratory for Novel Software Technology, Nanjing University \\ Collaborative Innovation Center of Novel Software Technology and Industrialization \\ Nanjing 210023, China \\ \{weit, liyf\}@lamda.nju.edu.cn
}

\begin{abstract}
Large-scale multi-label learning (LMLL) aims to annotate relevant labels from a large number of candidates for unseen data. Due to the high dimensionality in both feature and label spaces in LMLL, the storage overheads of LMLL models are often costly. This paper proposes a POP (joint label and feature Parameter OPtimization) method. It tries to filter out redundant model parameters to facilitate compact models. Our key insights are as follows. First, we investigate labels that have little impact on the commonly used LMLL performance metrics and only preserve a small number of dominant parameters for these labels. Second, for the remaining influential labels, we reduce spurious feature parameters that have little contribution to the generalization capability of models, and preserve parameters for only discriminative features. The overall problem is formulated as a constrained optimization problem pursuing minimal model size. In order to solve the resultant difficult optimization, we show that a relaxation of the optimization can be efficiently solved using binary search and greedy strategies. Experiments verify that the proposed method clearly reduces the model size compared to state-of-the-art LMLL approaches, in addition, achieves highly competitive performance.
\end{abstract}

\section{Introduction}

Large-scale multi-label learning (LMLL) (Zhang and Zhou 2014; Prabhu and Varma 2014) refers to annotate unseen data with the most relevant subset of labels from a large number of labels. It receives many applications recently. For example, in web page categorization (Partalas et al. 2015), there are thousands of labels (categories) from Wikipedia and one needs to annotate a new web page with its relevant labels; in image annotation (Deng et al. 2009), we have thousands of tags in the repository and one wishes to annotate each individual picture with its relevant tags. Similar observations can be found in some other applications, such as recommendation system (McAuley, Pandey, and Leskovec 2015), video classification (Abu-El-Haija et al. 2016) and so on.

LMLL suffers from the curse of dimensionality in both feature and label spaces (Jian et al. 2016; Niculescu-Mizil and Abbasnejad 2017). Normally, in LMLL applications, such as web page categorization and image annotation, data are represented by feature vectors with high dimensionality. On

Copyright (c) 2019, Association for the Advancement of Artificial Intelligence (www.aaai.org). All rights reserved.

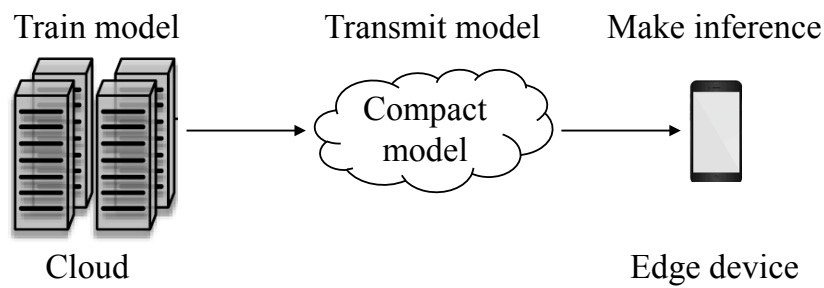

(a)

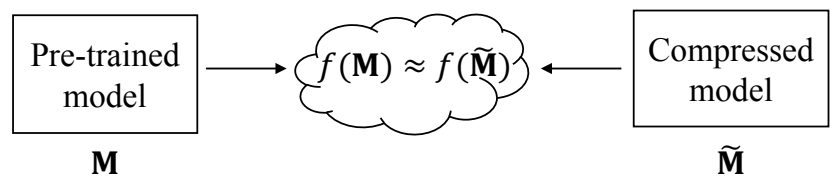

(b)

Figure 1: (a): In practice, a popular approach is to train a complete model for LMLL data with the usage of powerful computational platform, and then compress the model which is transmitted to edge devices to make inference. (b): The main difficulty in model compression is to retain a comparable performance while achieving a highly compressed model. Function $f$ measures the performance of the model.

the aspect of label space, data sets with thousands of labels have widely appeared in domains like object recognition and text classification (Zubiaga 2012).

The high dimensionality in both the feature and label spaces significantly increases the memory storage requirements, limiting the deployment of LMLL algorithms in realworld applications (Prabhu and Varma 2014). For example, many effective approaches of traditional multi-label learning, such as the popular binary relevance scheme (Tsoumakas, Katakis, and Vlahavas 2009; Zhang and Zhou 2014; Babbar and Schölkopf 2017; 2018), are hard to deal with modern LMLL data sets, since they have to systematically train a binary classifier for each label and thus the storage overhead scales linear to the number of features and labels, which is very expensive.

In this paper, we propose the POP (joint label and feature Parameter OPtimization) method to alleviate the storage overhead problem. Given a pre-trained model $\mathbf{M}$, the goal of our proposal is to minimize its model size without sig- 


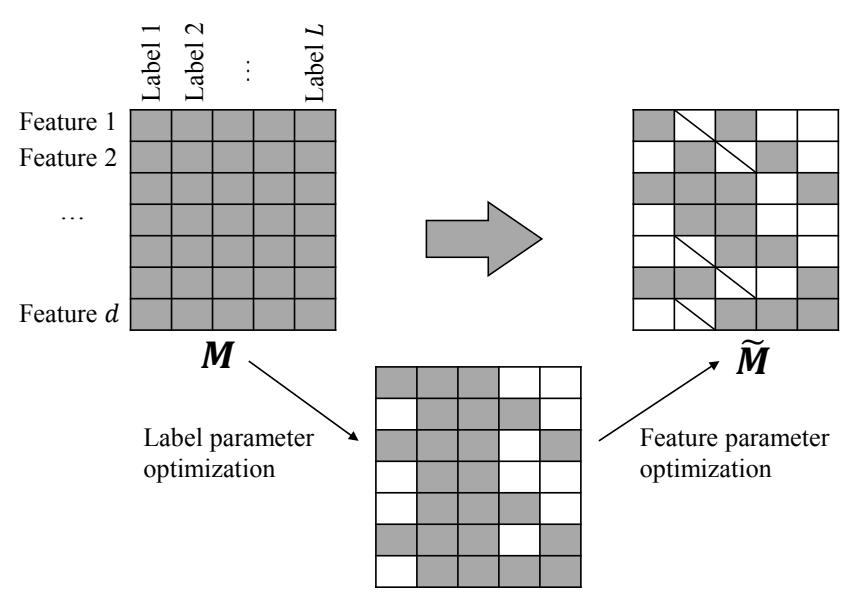

Pruned label parameter $\square$ Pruned feature parameter

Figure 2: An illustration of our method. Given a pre-trained model $\mathbf{M}$, we perform joint label and feature parameter optimization to facilitate a compact model $\tilde{\mathbf{M}}$.

nificantly losing predictive performance (as demonstrated in Figure 1a and Figure 1b). We illustrate the proposed model compression method in Figure 2.

- In terms of label parameter optimization, ideally the most influential label parameters in terms of LMLL metrics (PSP@ $k$ and PSnDCG@ $k$ ) are detected to facilitate compact models, which unfortunately is a hard optimization problem. Alternatively, we propose to detect the influential labels by calculating the performance impact for commonly used LMLL metrics. The analyses show that the performance impact of labels is closely related to the label importance and label frequency. Therefore, we only preserve a small number of dominant parameters for the labels that have little performance impact, which facilitates the compactness and retains a comparable performance.

- In terms of feature parameter optimization, since the most discriminative information is usually carried by only a subset of relevant features (Liu and Motoda 2007), we eliminate the noisy, redundant and irrelevant features which marginally affect the learning performance. Specifically, the spurious feature parameters which have little contribution to the generalization capability are removed, so that the model size is shrunk.

Generally, we formulate the above aspects as a constrained optimization problem pursuing minimal model size. In order to solve the resultant difficult optimization, we show that a relaxation of the optimization can be efficiently solved using binary search and greedy strategies. Experiments verify that the proposed method clearly reduces the model size compared to state-of-the-art LMLL approaches, in addition, achieves highly competitive performance.

In the following, we first introduce related work and the commonly used LMLL performance metrics, and then we present the POP method, with the experimental results. Finally we conclude this work.

\section{Related Work}

This work is mostly related to three branches of studies.

LMLL Model Compression Previous studies on LMLL model compression typically work on embedding approaches that project label vectors onto a low dimensional space based on the assumption that the label matrix is low-rank (Chen and Lin 2012; Kapoor, Viswanathan, and Jain 2012; Lin et al. 2014; Xu, Tao, and Xu 2016; Yeh et al. 2017). However, these studies do not take the pre-trained 'optimal' model into account and may lead to suboptimal performance. Recently, there are a few studies that yield a sparse version of the pretrained 'optimal' model by filtering out spurious features parameters (Babbar and Schölkopf 2017). However, they assume that all the labels are equally important, which may be not the case for LMLL performance metrics.

LMLL Feature Selection There are some studies about multi-label feature selection. For example, Zhang, Peña, and Robles (2009) adapted the classical naive Bayes classifiers. Ma et al. (2012) proposed to learn a feature subspace that is shared among multiple different classes. Jian et al. (2016) introduced a principled way of exploiting label correlations for feature selection in the presence of noisy and incomplete label information. All these studies assume that features are useful or useless for all labels. In many cases, however, one feature which is useless to some labels may be critical to some others. Direct multi-label feature selection typically obtains a suboptimal solution.

LMLL Label Selection There are also some proposals on label selection in LMLL. For example, Boutsidis, Mahoney, and Drineas (2009) proposed to find approximate solutions of the column subset selection problem efficiently. Bi and Kwok (2013) selected a small subset of labels that can approximately span the original label space. Weston, Makadia, and Yee (2013) partitioned the input space so that any given example can be mapped to a partition or set of partitions. Recently, Niculescu-Mizil and Abbasnejad (2017) proposed a label filter method to reduce prediction time. Different from above label selection methods, our proposal does not remove any label so that it can make predictions for all labels.

To our best knowledge, this paper is the first proposal on both label and feature parameter optimization for LMLL model compression.

\section{Common Performance Metrics in LMLL}

In this section, we introduce two commonly used LMLL performance metrics, PSP@ $k$ and PSnDCG@ $k$ (Babbar and Schölkopf 2018; Jain, Prabhu, and Varma 2016).

PSP@ $k$ The first one is Propensity Scored Precision@ $k$ (PSP@k) proposed in (Jain, Prabhu, and Varma 2016). PSP@ $k$ is popularly used in LMLL applications, especially for ranking tasks such as information retrieval. In PSP@ $k$, only a few top predictions of an instance will be considered. For instance $\mathbf{x} \in \mathbb{R}^{d}$ where $d$ represents the feature dimensionality, the PSP@ $k$ is defined for a predicted score vector 
$\hat{\mathbf{y}} \in \mathbb{R}^{L}$ and ground truth label vector $\mathbf{y} \in\{0,1\}^{L}$ as

$$
\operatorname{PSP} @ k(\mathbf{y}, \hat{\mathbf{y}}):=\frac{1}{k} \sum_{l \in \operatorname{rank}_{k}(\hat{\mathbf{y}})} \frac{\mathbf{y}_{l}}{p_{l}}
$$

where $L$ is the size of label set and $\operatorname{rank}_{k}(\hat{\mathbf{y}})$ returns the indices of $k$ largest value in $\hat{\mathbf{y}}$ ranked in descending order. $p_{l}=\frac{1}{1+C\left(N_{l}+B\right)^{-A}}$ is the propensity score for the $l$-th label, where $A, B, C$ are set in a heuristic manner on different data sets and $N_{l}$ is the number of the positive training instances.

PSnDCG@ $k$ Propensity Scored nDCG@ $k$ (PSnDCG@ $k$ ) is another commonly used ranking based performance measure in LMLL and is defined as

$$
\begin{array}{r}
\text { PSnDCG@ } k(\mathbf{y}, \hat{\mathbf{y}}):=\frac{\text { PSDCG@ } k}{\sum_{l=1}^{\min \left(k,\|\mathbf{y}\|_{0}\right)} \frac{1}{\log (l+1)}} \\
\text { where PSDCG@ } k(\mathbf{y}, \hat{\mathbf{y}}):=\sum_{l \in \operatorname{rank}_{k}(\hat{\mathbf{y}})} \frac{\mathbf{y}_{l}}{p_{l} \log (l+1)}
\end{array}
$$

In particular, when setting $p_{l}=1$ to all labels, PSP@ $k$ and PSnDCG@ $k$ reduce to another two popular LMLL performance metrics P@ $k$ and nDCG@ $k$, respectively.

\section{Joint Label and Feature Parameter Optimization (POP)}

In this section, we present the proposed framework POP. Given a pre-trained LMLL model, our goal is to minimize the model size while retaining a comparable performance with the pre-trained LMLL model.

Formally, given the pre-trained model $\mathbf{M} \in \mathbb{R}^{d \times L}$ and a set of $N$ samples $\mathcal{D}=\left\{\left(\mathbf{x}_{i}, \mathbf{y}_{i}\right)\right\}_{i=1}^{N}$ where $\mathbf{x}_{i} \in \mathbb{R}^{d}, \mathbf{y}_{i} \in$ $\{0,1\}^{L}$, the goal is to find a compact model $\tilde{\mathbf{M}}$ with comparable performance. Such objective can be formalized as,

$$
\begin{array}{ll}
\min _{\tilde{\mathbf{M}}} & \operatorname{size}(\tilde{\mathbf{M}}) \\
\text { s.t. } & f(\tilde{\mathbf{M}}, \mathcal{D}) \geq q^{*}-\epsilon
\end{array}
$$

where $\operatorname{size}(\tilde{\mathbf{M}})$ returns the model size of $\tilde{\mathbf{M}}, q^{*}$ is the performance of model $\mathbf{M}$ on $\mathcal{D}, \epsilon$ controls the tolerance to performance deterioration, and function $f$ measures the performance of model $\tilde{\mathbf{M}}$ on data $\mathcal{D}$. In this paper, the pre-trained model $\mathbf{M}$ is realized by binary relevance approaches shown state-of-the-art performance (Babbar and Schölkopf 2017; 2018; Niculescu-Mizil and Abbasnejad 2017).

More specifically, by formulating the model size of $\tilde{\mathbf{M}}$ as $\|\tilde{\mathbf{M}}\|_{0}$ and $f(\tilde{\mathbf{M}}, \mathcal{D})=\operatorname{perf}(\mathbf{X} \tilde{\mathbf{M}}, \mathbf{Y})$, where perf refers to commonly used LMLL performance metrics, i.e., PSP@ $k$ andPSnDCG@k. Eq. (1) can be reformulated as

$$
\begin{aligned}
& \min _{\tilde{\mathbf{M}}}\|\tilde{\mathbf{M}}\|_{0} \\
& \text { s.t. } \operatorname{perf}(\mathbf{X} \tilde{\mathbf{M}}, \mathbf{Y}) \geq q^{*}-\epsilon
\end{aligned}
$$

However, the resultant optimization problem in Eq. (2) is difficult due to non-smoothness and non-convexity (Weston et al. 2003). To conquer the resultant difficult optimization, we propose to solve a relaxation of the problem, from label and feature parameter optimization aspects jointly.

\section{Parameter Optimization w.r.t. Label}

When optimizing label parameter, ideally the most influential labels with respect to LMLL metrics are located, meanwhile, the model does not lose the predictive capability for the remaining labels. Such ideal situation can be cast as the following form,

$$
\begin{aligned}
\min _{\tilde{\mathbf{M}}} & \sum_{j=1}^{L}\|\| \tilde{\mathbf{M}}:, j\left\|_{0}-\delta\right\|_{0} \\
\text { s.t. } & \operatorname{perf}(\mathbf{X} \tilde{\mathbf{M}}, \mathbf{Y}) \geq q^{*}-\epsilon \\
& \|\tilde{\mathbf{M}}:, j\|_{0} \geq \delta, \quad j=1, \ldots, L
\end{aligned}
$$

where $\tilde{\mathbf{M}}_{:, j}$ indicates the $j$-th column of $\tilde{\mathbf{M}}$, which corresponds to the parameters for the $j$-th label, and $\delta$ is a small constant indicating the least number of parameters need to be preserved for each label. From Eq. (3), we maintain the predictive capability for each label and meanwhile minimize the number of performance-influential labels.

Eq. (3), however, is a difficult integer programming problem. Note that Eq. (3) can be viewed as selecting the most performance-influential labels. Following such intuition, we present to compute the performance impact of labels in terms of LMLL metrics so as to derive an approximate solution.

To capture the performance impact of labels, we study how labels affect the LMLL metrics under these two scenarios, i.e., missing labels and misclassified labels. The analysis shows that the performance impact of labels is proportional to its weight in LMLL metrics and its frequency in the observed data, which consequently provides a guideline to select the most influential labels.

Randomly Missing Labels Missing labels are commonly occurred in LMLL (Bi and Kwok 2013; Lin et al. 2014; $\mathrm{Xu}$, Tao, and $\mathrm{Xu} 2016)$. In this section, we formally capture the impact of labels under the missing labels scenario, that is, relevant labels are randomly missing with a probability $\pi$ (Lim, McAuley, and Lanckriet 2015). Without loss of generality, we let $u_{j}=\left\|\mathbf{Y}_{:, j}\right\|_{0}(j=1, \ldots, L)$ denote the number of instances that have the $j$-th label. We use $w_{j}=\frac{1}{p_{j}}$ to denote the weight for the $j$-th label, and $c_{i}$ indicating the number of relevant labels for instance $\mathbf{x}_{i}$.

Theorem 1. Suppose that relevant labels are randomly missing with probability $\pi$, the impact of the $j$-th label in terms of PSP@k and PSnDCG@ $k$ is upper bounded by $(1-\pi) w_{j} u_{j}$.

Proof. For PSP@ $k$, since $k$ of $c_{i}$ relevant labels are selected in the calculation of PSP@ $k$, we have $\left(\begin{array}{c}c_{i} \\ k\end{array}\right)$ distinct ways. The expected influence of the $j$-th label is computed as,

$$
\frac{w_{j}}{k} \sum_{i=1 \wedge \mathbf{Y}_{i j}=1}^{N} \frac{\left(\begin{array}{c}
c_{i}-1 \\
k-1
\end{array}\right)}{\left(\begin{array}{c}
c_{i} \\
k
\end{array}\right)}=(1-\epsilon) \sum_{i=1 \wedge \mathbf{Y}_{i j}=1}^{N} \frac{w_{j}}{c_{i}} \leq(1-\pi) w_{j} u_{j}
$$

It can be seen that the impact of the $j$-th label is upper bounded by the product of label weight $w_{j}$, its frequency $u_{j}$ and a constant.

For PSnDCG@ $k$, note that every observed label has the same rank, hence $r=\frac{1}{\log (l+1)}$, for $l=\{1, \ldots, k\}$, is a 
constant and we have

$$
\begin{aligned}
\text { PSnDCG@ } k & =\frac{\text { PSDCG@ } k}{\sum_{l=1}^{\min \left(k,\|\mathbf{y}\|_{0}\right)} \frac{1}{\log (l+1)}} \\
& =\frac{r}{\sum_{l=1}^{\min \left(k,\|\mathbf{y}\|_{0}\right)} r} \sum_{l \in \operatorname{rank}_{k}(\hat{\mathbf{y}})} \mathbf{y}_{l}
\end{aligned}
$$

Since $L$ is large in LMLL and $k \leq 5$, we usually have $\|\mathbf{y}\|_{0} \geq k$ and PSnDCG@ $k$ is cast as follows.

$$
\begin{aligned}
\text { PSnDCG@ } k & =\frac{r}{\sum_{l=1}^{k} r} \sum_{l \in \operatorname{rank}_{k}(\hat{\mathbf{y}})} \mathbf{y}_{l} \\
& =\frac{1}{k} \sum_{l \in \operatorname{rank}_{k}(\hat{\mathbf{y}})} \mathbf{y}_{l}=\text { PSP@k}
\end{aligned}
$$

As a result, the analysis for PSnDCG@ $k$ is reduced to the oneinPSP@k.

Randomly Misclassified Labels Another common scenario is label misclassification (Schapire 1990; Wei and Li 2018). We also compute the impact of labels under the misclassified label scenario, that is, labels are randomly misclassified with probability $\pi$.

Theorem 2. Suppose that labels are randomly misclassified with probability $\pi$, the impact of the $j$-th label on PSP@k and PSnDCG@k is upper bounded by $\frac{(1-\pi)}{(L-2) \pi+1} w_{j} u_{j}$.

Proof. For PSP@ $k$, there are $v_{i}=(1-\pi) c_{i}+\pi\left(L-c_{i}\right)$ relevant labels in the predicted label vector. By choosing a random subset of size $k$ from $v_{i}$ labels, the influence of the $j$-th label to PSP@ $k$ can be computed as

$$
\frac{w_{j}}{k} \sum_{i=1 \wedge \mathbf{Y}_{i j}=1}^{N} \frac{\left(\begin{array}{c}
v_{i}-1 \\
k-1
\end{array}\right)}{\left(\begin{array}{c}
v_{i} \\
k
\end{array}\right)}=\sum_{i=1 \wedge \mathbf{Y}_{i j}=1}^{N} \frac{w_{j}}{v_{i}} \leq \frac{(1-\pi) w_{j} u_{j}}{(L-2) \pi+1}
$$

Eq. (4) shows that the influence of the $j$-th label is upper bounded by $\frac{(1-\pi)}{(L-2) \pi+1} w_{j} u_{j}$.

For PSnDCG@ $k$, similar with the proof in Theorem 1, the proof for PSnDCG@ $k$ is reduced to the one in PSP@ $k$, and we obtain same conclusive remark for PSnDCG@ $k$.

According to the analyses, in both label-missing and labelmisclassified scenarios, we find that impact of labels in terms of commonly LMLL metrics (PSP@ $k$ and PSnDCG@ $k$ ) is proportional to the product of label weights and label frequencies. Therefore, we rank the labels according to the value of $w_{j} u_{j}, j=\{1, \ldots, L\}$ in ascending order, and filter out parameters for labels with little performance-impact. Due to the large number of labels, it is expensive to remove parameters label by label until the constraint is violated. To this end, a binary search is developed to efficiently determine the threshold based on the observation that the performance is monotonically decreasing as the number of removed label parameters increases. By doing this, the computational cost is reduced from $O(L)$ to $O(\log L)$. Algorithm 1 summarizes the detailed procedure of binary search for label parameter optimization.

\section{Parameter Optimization w.r.t. Feature}

We further locate the most important feature parameters for the influential labels detected from label parameter optimization, and consequently remove spurious feature parameters to facilitate a compact model. Considering that Eq. (2) is difficult to solve due to non-smoothness and non-convexity, to this end, we propose to solve a relaxation of Eq. (2),

$$
\begin{aligned}
& \min _{\tilde{\mathbf{M}}}\left\|\tilde{\mathbf{Y}}-\mathbf{Y}^{*}\right\|_{F}^{2}+\lambda\|\tilde{\mathbf{M}}\|_{0} \\
& \text { s.t. } \tilde{\mathbf{Y}}=\mathbf{X} \mathbf{\mathbf { M }} ; \mathbf{Y}^{*}=\mathbf{X M}
\end{aligned}
$$

where the constraint $\operatorname{perf}(\mathbf{X} \tilde{\mathbf{M}}, \mathbf{Y}) \geq q *-\epsilon$ in Eq. (2) is relaxed as $\left\|\mathbf{Y}-\mathbf{Y}^{*}\right\|_{F}^{2}$, which encourages the predicted label matrix $\tilde{\mathbf{Y}}$ of $\tilde{\mathbf{M}}$ and $\mathbf{Y}^{*}$ from the pre-trained model $\mathbf{M}$ to be closely related. The second term in Eq. (5) minimizes the number of non-zero entries in $\tilde{\mathbf{M}}$, and the hyper-parameter $\lambda>0$ trades off the predictive accuracy and the model size.

The above optimization is difficult to solve. Inspired by (Zhao and Yu 2006), an approximate solution can be obtained by setting feature parameters that lie in range $[-\sqrt{\lambda}, \sqrt{\lambda}]$ to 0 . The closed-form approximation is based on the observation that each time a model parameter is weeded out, the term $\lambda|| \tilde{\mathbf{M}} \|_{0}$ decreases by $\lambda$ irrespective of its value. Meanwhile, to minimize the reconstruction error $\left\|\mathbf{Y}-\mathbf{Y}^{*}\right\|_{F}^{2}$, we filter out model parameters starting from the entries with small absolute values to entries with large absolute values. The procedure terminates when the absolute value of the entry that is to be removed in the next round is greater than $\sqrt{\lambda}$, which results in an increase to the objective. The pseudocode of the POP algorithm is summarized in Algorithm 2.

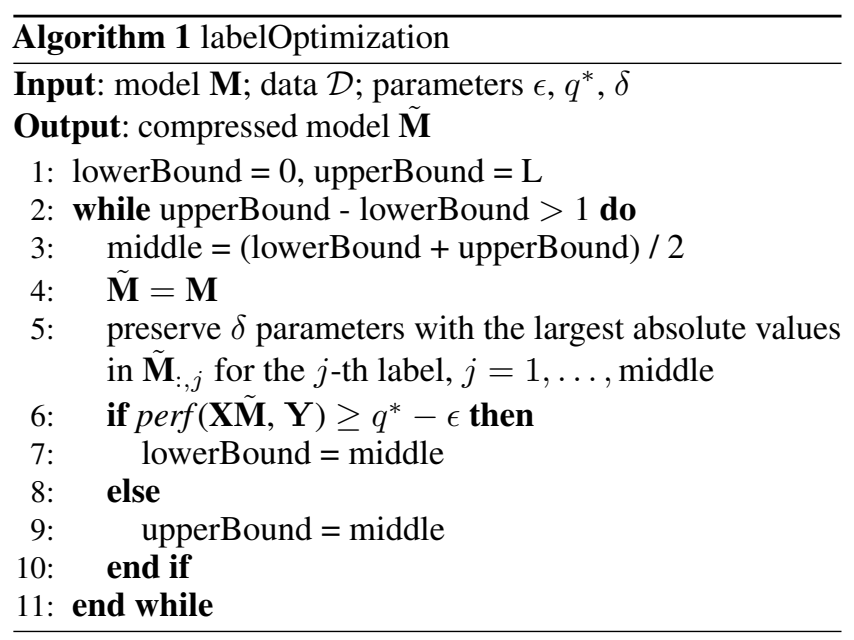

\section{Experiments}

We carry out extensive experiments on LMLL benchmark data sets to evaluate the effectiveness of our proposal.

\section{Experimental Setup}

Data sets Experiments are conduct on benchmark data sets bibtex (text categorization, 159 labels), delicious (image annotation, 983 labels), eurlex (text categorization, 3993 labels) 


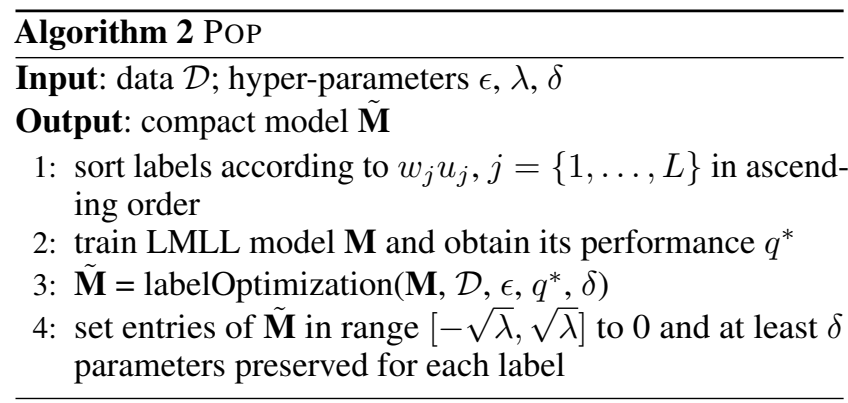

and wiki10 (web page categorization, 30K labels). We report and compare the results using the same train/test splits of data sets. All the data sets as well as the experimental results of state-of-the-art LMLL methods are publicly available, and can be downloaded from the Extreme Classification Repository ${ }^{1}$.

Compared Methods We compare our method to Binary Relevance (BR) and six state-of-the-art LMLL baselines.

- Binary Relevance (Zhang and Zhou 2014) builds one-vsall SVM for each label using Liblinear (Fan et al. 2008).

- LEML (Yu et al. 2014) is an embedding method based on low-rank empirical risk minimization.

- FastXML (Prabhu and Varma 2014) is a random forest based LMLL approach.

- SLEEC (Bhatia et al. 2015) learns the embedding of labels by preserving the pairwise distances between a few nearest label neighbors.

- $\mathrm{CoH}$ (Shen et al. 2018) proposes a co-hashing method which jointly compresses the input and output into compact binary embeddings.

- DisMEC (Babbar and Schölkopf 2017) learns a 1vsA linear-SVM in a distributed fashion.

- PD-Sparse (Yen et al. 2016) proposes to solve $L_{1}$ regularized multi-class loss using Frank-Wolfe based algorithm.

We use BR as the base classifier and build POP based on it.

Hyper-parameters In all of our experiments, we fix the least number of preserved label parameters $\delta$ to 5. For LEML, FastXML, SLEEC, and $\mathrm{CoH}$, we use the default parameter settings in the code.

Computational Device All experimental comparisons are conducted on a same PC machine with an Intel i5-6500 3.20GHz CPU and 32GB RAM.

\section{PoP vs. Uncompressed Baseline}

We first study how effective POP is at reducing the model size in comparison with plain BR. The comparison results with the plain binary relevance approach are depicted in Table 1. On relatively small data set bibtex, PoP improves above $50 \%$ model size and loses no more than $0.5 \%$ performance in terms of six different metrics. Considering the relatively balanced label distribution due to the small label set and hence only a

\footnotetext{
${ }^{1}$ http://manikvarma.org/downloads/XC/XMLRepository.html
}

few label parameters can be pruned, otherwise resulting in serious performance deterioration. On the other three larger data sets with high dimensionality of feature and label space, the model size is improved by more than $80 \%$. As a result, we obtain a significant reduction in model size with highly comparable generalization performance.

\section{PoP vs. Compressed Baselines}

We further compare the performance of POP with DiSMEC and PD-Saprse. We report the result from the Extreme Classification Repository in Table 2. DiSMEC reduces the model size by pruning spurious feature parameters as a postprocessing step, which can be viewed as a subprocedure of POP. In terms of PSP@ $k$ and PSnDCG@ $k$, DiSMEC achieves similar performance with our method. However, for model size, PoP leads to as much as $10 x$ reduction on wiki10 and about $4 \mathrm{x}$ reduction on eurlex, which shows the merit of our proposal. For PD-Sparse, due to its linear nature, its model size is small, but predictive accuracy is also limited by the capacity of the model. For instance, PoP gets more than $5 \%$ better performance than PD-Sparse in most cases. The result shows that POP finds a proper balance between model capacity and predictive accuracy.

\section{PoP vs. State-of-the-art Methods}

In this experiment, we compare the performance of POP with state-of-the-art methods: FastXML, LEML, SLEEC, and $\mathrm{CoH}$. As demonstrated in Table 2, although PoP is built on the binary relevance scheme, it achieves comparable or even smaller model size compared to state-of-the-art approaches. However, in terms of predictive performance, solvers relied on structural assumptions such as FastXML (tree), LEML (low-rank), SLEEC (piecewise-low-rank) do not perform as well as POP in most cases. This may owe to the fact that lowrank or tree assumption does not exactly hold in these data sets. On the aspect of model size, we can see that PoP gets an order of magnitude smaller model size than FastXML and SLEEC. Compared with $\mathrm{CoH}$, we achieve better performance with a large margin on all data sets and a smaller model size in most cases.

\section{Parameter Sensitivities Analysis}

We further investigate the influence of $\epsilon$ and $\lambda$ to the performance of POP.

Impact of $\epsilon$ We study how different values of $\epsilon$ impact the predictive accuracy and model size. Figure 3 demonstrates that performance deteriorates as the value of $\epsilon$ grows because it determines the fraction of removed parameters for labels and in return, hurts the performance when informative label parameters are pruned. On the aspect of model size, POP is able to filter out more than $80 \%$ model parameters even when $\epsilon=1 \%$. Although more significant reduction can be gained with a larger value of $\epsilon$, it comes at the cost of losing generalization accuracy.

Impact of $\lambda$ Figure 4 shows the performance and model size is a function of the value of $\lambda$ which trades off model size and the predictive performance. We can see that when $\lambda$ is 
Table 1: Performance comparison between the proposed PoP method and BR in terms of PSP@ $k(\%)$, PSnDCG@ $k(\%)$, and Model size with $\lambda=0.01$ and $\epsilon=1 \%$. The best results in terms of model size are in bold.

\begin{tabular}{cc|ccccccc}
\hline Data set & & PSP@1 & PSP@3 & PSP@5 & PSnDCG@1 & PSnDCG@3 & PSnDCG@5 & Model size \\
\hline \multirow{2}{*}{ bibtex } & BR & 50.70 & 53.66 & 59.34 & 50.70 & 52.71 & 55.80 & $1.15 \mathrm{MB}$ \\
& POP & 50.71 & 53.30 & 58.86 & 50.71 & 52.39 & 55.41 & $\mathbf{0 . 5 9}$ MB \\
\hline \multirow{2}{*}{ delicious } & BR & 32.14 & 33.59 & 33.43 & 32.14 & 33.32 & 33.28 & $7.18 \mathrm{MB}$ \\
& POP & 32.08 & 33.59 & 33.47 & 32.08 & 33.30 & 33.29 & $\mathbf{1 . 2 6}$ MB \\
\multirow{2}{*}{ eurlex } & BR & 39.93 & 45.86 & 49.74 & 39.93 & 44.24 & 46.83 & $156.38 \mathrm{MB}$ \\
& POP & 40.06 & 46.02 & 49.91 & 40.06 & 44.42 & 47.01 & $\mathbf{2 0 . 1 8}$ MB \\
\hline \multirow{2}{*}{ wiki10 } & BR & 13.57 & 13.10 & 13.96 & 13.60 & 13.82 & 13.97 & $23.50 \mathrm{~GB}$ \\
& POP & 13.53 & 13.10 & 13.46 & 13.53 & 13.65 & 13.67 & $\mathbf{6 7 . 5 0} \mathbf{M B}$ \\
\hline
\end{tabular}

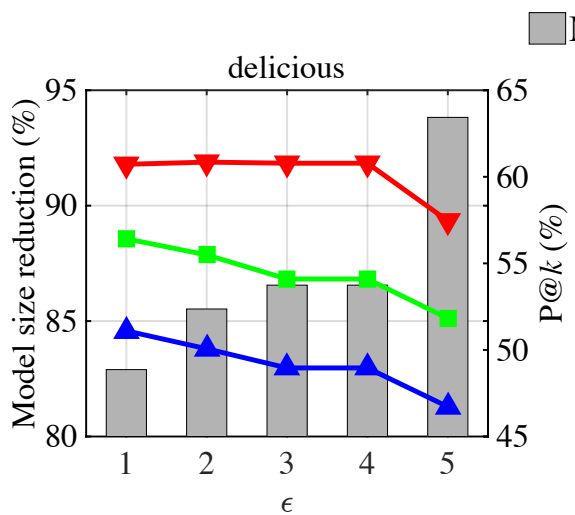

Model size reduction-P@1-P@3 $=\mathrm{P} @ 5$
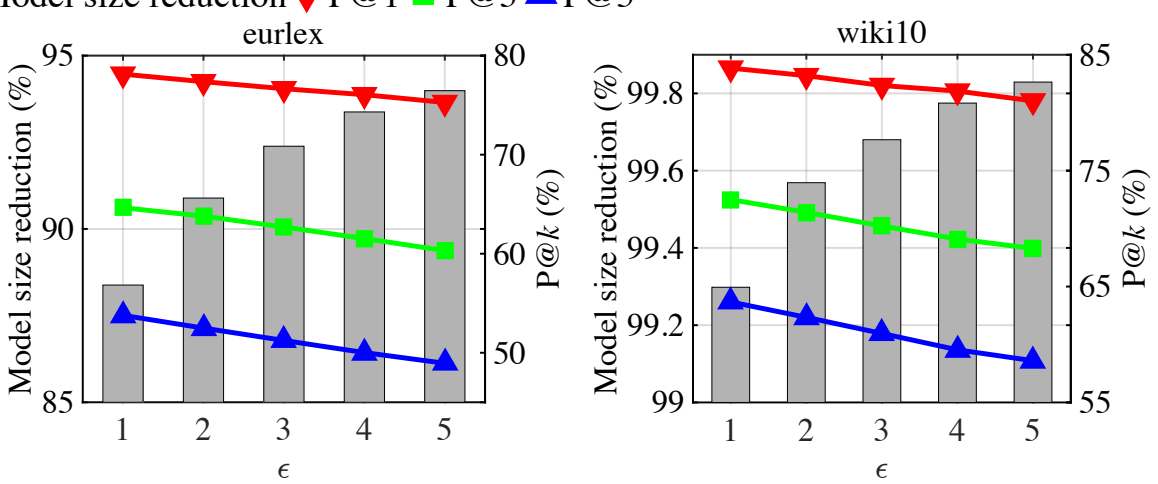

Figure 3: Study on different value of $\epsilon$ with $\lambda$ set to 0.01 . X-axis: value of $\epsilon(\%)$. Y-axis (Left): percentage of model size reduction compared to the plain BR. Y-axis (Right): P@ $k$.

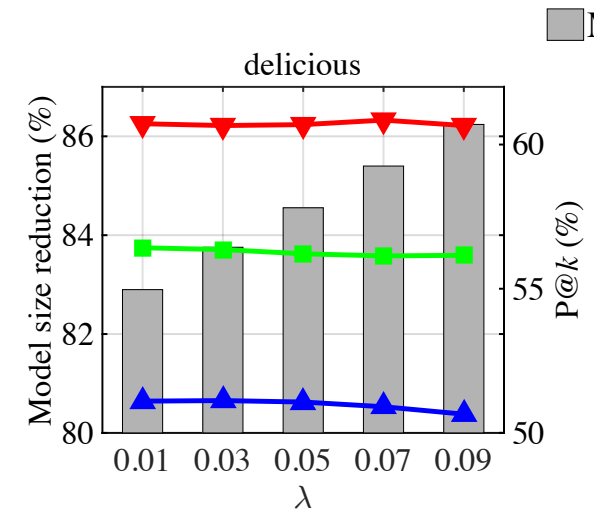

Model size reduction־P@1=-P@3 $0 \mathrm{P} @ 5$
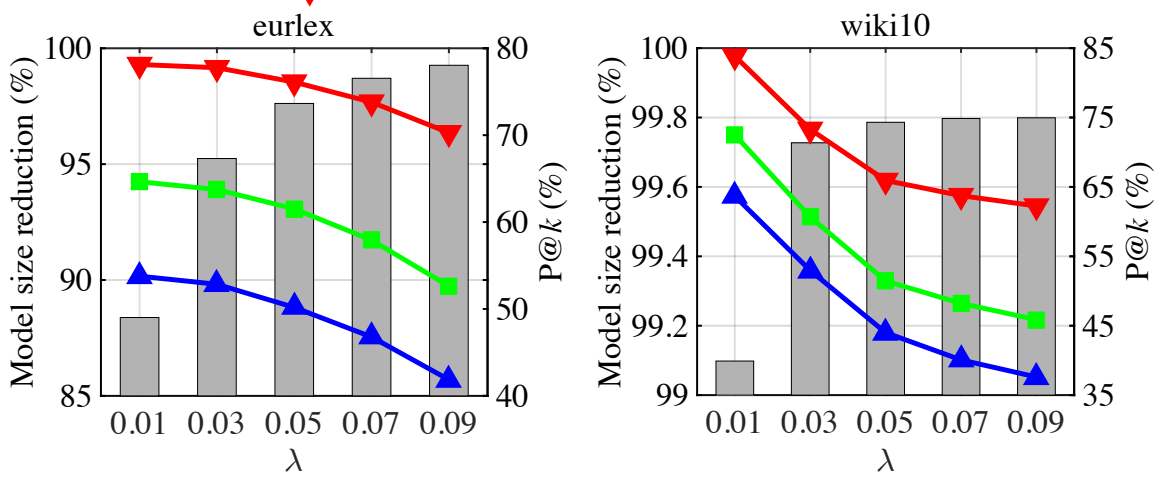

Figure 4: Study on different value of $\lambda$ with $\epsilon$ set to $1 \%$. X-axis: value of $\lambda$. Y-axis (Left): percentage of model size reduction compared to the plain BR. Y-axis (Right): P@ $k$.

small, the model size is reduced by at least $80 \%$. As the value of $\lambda$ slightly grows, the performance does not affect. However, when too large values of $\lambda$ are used, the model becomes excessively sparse and many discriminative parameters are wrongly eliminated, deteriorating performance. Our empirical results show that POP performs well in a wide range of $\epsilon$, but is relatively sensitive to $\lambda$ on some data sets.

\section{Conclusion}

In this work, we propose a simple and powerful approach to compress model size for LMLL by the joint label and feature parameter optimization. Our key insight is to weed out label 
Table 2: Comparison with state-of-the-art approaches in terms of model size, PSP@ $k(\%)$ and PSnDCG@ $k$ (\%). Results for $\mathrm{CoH}$ are partially available due to the high computational cost. The best and the second best results are in bold.

\begin{tabular}{|c|c|c|c|c|c|c|c|c|}
\hline Data set & & FastXML & LEML & SLEEC & $\mathrm{CoH}$ & DiSMEC & PD-Sparse & POP (ours) \\
\hline \multirow{7}{*}{ bibtex } & Model size & $18.72 \mathrm{MB}$ & $0.76 \mathrm{MB}$ & $2.46 \mathrm{MB}$ & $4.70 \mathrm{MB}$ & $0.71 \mathrm{MB}$ & $20.00 \mathrm{~KB}$ & $0.59 \mathrm{MB}$ \\
\hline & PSP@1 & 48.54 & 47.97 & 51.12 & 36.53 & 50.20 & 48.34 & 50.71 \\
\hline & PSP@3 & 52.30 & 51.42 & 53.95 & 28.20 & 52.20 & 48.77 & 53.30 \\
\hline & PSP@5 & 58.28 & 57.53 & 59.56 & 25.59 & 58.60 & 52.93 & 58.86 \\
\hline & PSnDCG@1 & 48.54 & 47.97 & 51.12 & 36.58 & 50.20 & 48.34 & 50.71 \\
\hline & PSnDCG@3 & 51.11 & 50.25 & 52.99 & 30.62 & 52.00 & 48.49 & 52.39 \\
\hline & PSnDCG@5 & 54.38 & 53.59 & 56.04 & 29.06 & 55.70 & 50.72 & 55.41 \\
\hline \multirow{7}{*}{ delicious } & Model size & $71.29 \mathrm{MB}$ & $2.26 \mathrm{MB}$ & $7.34 \mathrm{MB}$ & $10.92 \mathrm{MB}$ & - & $0.25 \mathrm{MB}$ & $1.26 \mathrm{MB}$ \\
\hline & PSP@1 & 32.35 & 30.73 & 32.11 & 20.43 & - & 25.22 & 32.08 \\
\hline & PSP@3 & 34.51 & 32.43 & 33.21 & 22.76 & - & 24.63 & 33.59 \\
\hline & PSP@5 & 35.43 & 33.26 & 33.83 & 24.11 & - & 23.85 & 33.47 \\
\hline & PSnDCG@1 & 32.35 & 30.73 & 32.11 & 20.43 & - & 25.22 & 32.08 \\
\hline & PSnDCG@3 & 34.00 & 32.01 & 32.93 & 22.16 & - & 24.80 & 33.30 \\
\hline & PSnDCG@5 & 34.73 & 32.66 & 33.41 & 23.20 & - & 24.25 & 33.29 \\
\hline \multirow{7}{*}{ eurlex } & Model size & $194.40 \mathrm{MB}$ & $34.31 \mathrm{MB}$ & $245.49 \mathrm{MB}$ & 15.95 MB & $79.86 \mathrm{MB}$ & $25.00 \mathrm{MB}$ & 20.18 MB \\
\hline & PSP@1 & 26.62 & 24.10 & 34.25 & 20.78 & 41.20 & 38.28 & 40.06 \\
\hline & PSP@3 & 34.16 & 27.20 & 39.83 & 22.98 & 45.40 & 42.00 & 46.02 \\
\hline & PSP@5 & 38.96 & 29.09 & 42.76 & 21.89 & 49.30 & 44.89 & 49.91 \\
\hline & PSnDCG@1 & 26.62 & 24.10 & 34.25 & 20.78 & 41.20 & 38.28 & 40.06 \\
\hline & PSnDCG@3 & 32.07 & 26.37 & 38.35 & 22.49 & 44.30 & 40.96 & 43.55 \\
\hline & PSnDCG@5 & 35.23 & 27.62 & 40.30 & 21.92 & 46.90 & 42.84 & 47.01 \\
\hline \multirow{7}{*}{ wiki10 } & Model size & 501.47 MB & $506.88 \mathrm{MB}$ & $924.60 \mathrm{MB}$ & - & $880.00 \mathrm{MB}$ & - & 67.50 MB \\
\hline & PSP@1 & 9.80 & 9.41 & 11.14 & - & 13.60 & - & 13.53 \\
\hline & PSP@3 & 10.17 & 10.07 & 11.86 & - & 13.10 & - & 13.10 \\
\hline & PSP@5 & 10.54 & 10.55 & 12.40 & - & 13.80 & - & 13.46 \\
\hline & PSnDCG@1 & 9.80 & 9.41 & 11.14 & - & 13.60 & - & 13.53 \\
\hline & PSnDCG@3 & 10.08 & 9.90 & 11.68 & - & 13.20 & - & 13.65 \\
\hline & PSnDCG@5 & 10.33 & 10.24 & 12.06 & - & 13.60 & - & 13.67 \\
\hline
\end{tabular}

parameters which have little impact on the predictive accuracy, as well as to prune redundant feature parameters. We formulate this as a constrained optimizing problem and solve its relaxation form effectively with binary search and greedy strategies. Empirical results demonstrate that the proposed method is capable of reducing the model size, in addition, achieves highly competitive performance. In future, we will study how to improve the performance on few relevant instances (Wei et al. 2018) and derive LMLL models with both high performance and lightweight storage.

\section{Acknowledgments}

This research was supported by the National Key R\&D Program of China (2017YFB1001903) and the National Natural Science Foundation of China (61772262). Yu-Feng Li is the corresponding author. We also would like to thank Wei-Wei Tu and Hai Wang for helpful discussions.

\section{References}

Abu-El-Haija, S.; Kothari, N.; Lee, J.; Natsev, P.; Toderici, G.; Varadarajan, B.; and Vijayanarasimhan, S. 2016. Youtube8m: A large-scale video classification benchmark. arXiv preprint arXiv:1609.08675.

Babbar, R., and Schölkopf, B. 2017. Dismec: Distributed sparse machines for extreme multi-label classification. In Proceedings of the 10th ACM International Conference on Web Search and Data Mining, 721-729.

Babbar, R., and Schölkopf, B. 2018. Adversarial extreme multi-label classification. arXiv preprint arXiv:1803.01570.

Bhatia, K.; Jain, H.; Kar, P.; Varma, M.; and Jbabain, P. 2015. Sparse local embeddings for extreme multi-label classification. In Advances in Neural Information Processing Systems 28. 730-738.

Bi, W., and Kwok, J. T. 2013. Efficient multi-label classifi- 
cation with many labels. In Proceedings of the 30th International Conference on Machine Learning, 405-413.

Boutsidis, C.; Mahoney, M. W.; and Drineas, P. 2009. An improved approximation algorithm for the column subset selection problem. In Proceedings of the 20th annual ACMSIAM symposium on Discrete algorithms, 968-977.

Chen, Y.-N., and Lin, H.-T. 2012. Feature-aware label space dimension reduction for multi-label classification. In $A d$ vances in Neural Information Processing Systems 25. 15291537.

Deng, J.; Dong, W.; Socher, R.; Li, L.-J.; Li, K.; and F.-F., L. 2009. Imagenet: A large-scale hierarchical image database. In Proceedings of the 22nd IEEE Conference on Computer Vision and Pattern Recognition, 248-255.

Fan, R.-E.; Chang, K.-W.; Hsieh, C.-J.; Wang, X.-R.; and Lin, C.-J. 2008. Liblinear: A library for large linear classification. Journal of Machine Learning Research 9:1871-1874.

Jain, H.; Prabhu, Y.; and Varma, M. 2016. Extreme multilabel loss functions for recommendation, tagging, ranking $\&$ other missing label applications. In Proceedings of the 22nd ACM SIGKDD International Conference on Knowledge Discovery and Data Mining, 935-944.

Jian, L.; Li, J.-D.; Shu, K.; and Liu, H. 2016. Multi-label informed feature selection. In Proceedings of the 25th International Joint Conference on Artificial Intelligence, 16271633.

Kapoor, A.; Viswanathan, R.; and Jain, P. 2012. Multilabel classification using bayesian compressed sensing. In $A d$ vances in Neural Information Processing Systems 25. 26452653.

Lim, D.; McAuley, J.; and Lanckriet, G. 2015. Top-n recommendation with missing implicit feedback. In Proceedings of the 9th ACM Conference on Recommender Systems, 309312.

Lin, Z.-J.; Ding, G.-G.; Hu, M.-Q.; and Wang, J.-M. 2014. Multi-label classification via feature-aware implicit label space encoding. In Proceedings of the 31st International Conference on Machine Learning, 325-333.

Liu, H., and Motoda, H. 2007. Computational methods of feature selection. CRC Press.

Ma, Z.-G.; Nie, F.-P.; Yang, Y.; Uijlings, J. R.; and Sebe, N. 2012. Web image annotation via subspace-sparsity collaborated feature selection. IEEE Transactions on Multimedia 14(4):1021-1030.

McAuley, J.; Pandey, R.; and Leskovec, J. 2015. Inferring networks of substitutable and complementary products. In Proceedings of the 21th ACM SIGKDD International Conference on Knowledge Discovery and Data Mining, 785-794.

Niculescu-Mizil, A., and Abbasnejad, M. E. 2017. Label filters for large scale multi-label classification. In Proceedings of the 20th International Conference on Artificial Intelligence and Statistics, 1448-1457.

Partalas, I.; Kosmopoulos, A.; Baskiotis, N.; Artieres, T.; Paliouras, G.; Gaussier, E.; Androutsopoulos, I.; Amini, M.R.; and Galinari, P. 2015. Lshtc: A benchmark for large-scale text classification. arXiv preprint arXiv:1503.08581.
Prabhu, Y., and Varma, M. 2014. Fastxml: A fast, accurate and stable tree-classifier for extreme multi-label learning. In Proceedings of the 20th ACM SIGKDD International Conference on Knowledge Discovery and Data Mining, 263-272.

Schapire, R. E. 1990. The strength of weak learnability. Machine Learning 5(2):197-227.

Shen, X.; Liu, W.; Tsang, I. W.; Sun, Q.-S.; and Ong, Y.-S. 2018. Compact multi-label learning. In Proceedings of the 32nd AAAI Conference on Artificial Intelligence, 4066-4073.

Tsoumakas, G.; Katakis, I.; and Vlahavas, I. 2009. Mining multi-label data. In Data Mining and Knowledge Discovery handbook. Springer. 667-685.

Wei, T., and Li, Y.-F. 2018. Does tail label help for large-scale multi-label learning. In Proceedings of the 27th International Joint Conference on Artificial Intelligence, 2847-2853.

Wei, T.; Guo, L.-Z.; Li, Y.-F.; and Gao, W. 2018. Learning safe multi-label prediction for weakly labeled data. Machine Learning 107(4):703-725.

Weston, J.; Elisseeff, A.; Schölkopf, B.; and Tipping, M. 2003. Use of the zero-norm with linear models and kernel methods. Journal of Machine Learning Research 3:14391461.

Weston, J.; Makadia, A.; and Yee, H. 2013. Label partitioning for sublinear ranking. In Proceedings of the 30th International Conference on Machine Learning, 181-189.

Xu, C.; Tao, D.-C.; and Xu, C. 2016. Robust extreme multilabel learning. In Proceedings of the 22nd ACM SIGKDD International Conference on Knowledge Discovery and Data Mining, 1275-1284.

Yeh, C.-K.; Wu, W.-C.; Ko, W.-J.; and Wang, Y.-C. F. 2017. Learning deep latent space for multi-label classification. In Proceedings of the 31st AAAI Conference on Artificial Intelligence, 2838-2844.

Yen, I. E.-H.; Huang, X.-R.; Ravikumar, P.; Zhong, K.; and Dhillon, I. S. 2016. Pd-sparse: A primal and dual sparse approach to extreme multiclass and multilabel classification. In Proceedings of the 33rd International Conference on Machine Learning, 3069-3077.

Yu, H.-F.; Jain, P.; Kar, P.; and Dhillon, I. S. 2014. Largescale multi-label learning with missing labels. In Proceedings of the 31st International Conference on Machine Learning, 593-601.

Zhang, M.-L., and Zhou, Z.-H. 2014. A review on multi-label learning algorithms. IEEE Transactions on Knowledge and Data Engineering 26(8):1819-1837.

Zhang, M.-L.; Peña, J. M.; and Robles, V. 2009. Feature selection for multi-label naive bayes classification. Information Sciences 179(19):3218-3229.

Zhao, P., and Yu, B. 2006. On model selection consistency of lasso. Journal of Machine learning research 7:2541-2563.

Zubiaga, A. 2012. Enhancing navigation on wikipedia with social tags. arXiv preprint arXiv:1202.5469. 